\title{
Lamia e le sue metamorfosi
}

\author{
Chiara CAPPANERA \\ Università degli Studi di Genova \\ chiara_cappanera@libero.it
}

Recibido: 5 de noviembre de 2015

Aceptado: 19 de febrero de 2016

\section{RIASSUNTO}

Il lavoro analizza la figura di Lamia alla luce delle testimonianza testuali e iconografiche. L'obiettivo è quello di distinguerla dalle altre figure di demoni femminili presenti nel panorama greco dei demoni rapitori di bambini e di comprendere se la sua androginia sia un elemento originario o acquisito in seguito ai contatti culturali con il Vicino Oriente. Attraverso lo studio delle fonti è quindi possibile seguire questa figura e tutte le metamorfosi da lei subite, sia nella cultura greca sia in quella latina.

Parole Chiave: Lamia. Demoni rapitori. Pittore di Beldam. Androginia. Lemures.

\section{Lamia and her Metamorphosis}

\begin{abstract}
The present research analyzes Lamia's figure, thanks to literary and iconographic sources. The purpose is distinguishing Lamia from the other child-snatching female demons of Greek culture and understanding if her androgyny is an original element or if it is taken from the Near Middle East. Through the study of sources is possible to follow Lamia and all of her metamorphosis, in Greek culture and also in the Latin one.
\end{abstract}

Keywords: Lamia. Child-snatching demons. The Beldam Painter. Androgyny. Lemures. 
Nel variegato panorama delle figure mitologiche minori si annovera tutta una serie di demoni femminili, dotati di una scarsa individualità, dediti alla caccia e all'uccisione di bambini e neonati; tra questi spicca il personaggio di Lamia, creatura che si fatica a definire in toto 'femminile'. Nonostante sia presentata come un'amante di Zeus, tanto bella quanto sfortunata, perseguitata da Hera e indotta dal dolore a rapire i figli delle altre donne, nella commedia antica essa entra in scena dotata di attributi sessuali maschili.

Sicuramente essa è protagonista di un processo di metamorfosi. Tutti gli scoliasti, ${ }^{1}$ che si sentono in dovere di dare informazioni aggiuntive su questa figura, concordano nel ritenere che, da un certo momento in poi, Lamia abbia mutato forma; due su tre sostengono si sia trasformata in un mostro per il dolore provocato dalla morte, di cui sarebbe artefice lei stessa o Hera, dei figli, uno che il padre degli dèi le abbia concesso il dono di assumere altre sembianze in risarcimento di queste morti. Nessuno però parla di un cambio di genere; quei "sozzi testicoli" rimangono inspiegabili.

Bisogna chiedersi, avvalendosi anche delle possibili testimonianze iconografiche, quante 'lamie' siano confluite in Lamia e, se possibile, quale sia la forma originaria di questo personaggio, quali le sue caratteristiche e quale la sua storia.

Innanzitutto è necessario analizzare la prima attestazione cronologica della figura di Lamia; ${ }^{2}$ nelle Vespe si trovano i seguenti versi:

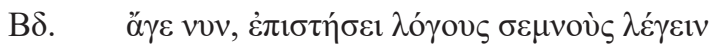

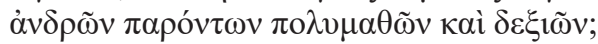

Ф.. $\ddot{\varepsilon} \gamma \omega \gamma \varepsilon$.

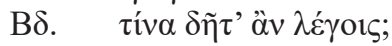

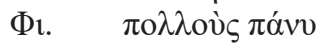

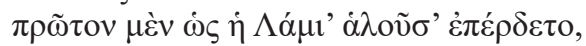

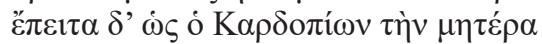

Il passo, così come si presenta, doveva essere poco comprendibile per il lettore di età bizantina; uno scoliasta ha infatti ritenuto necessario apporre a margine una picco-

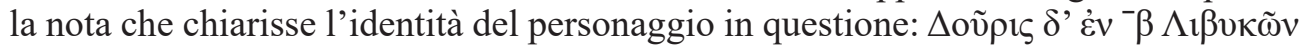

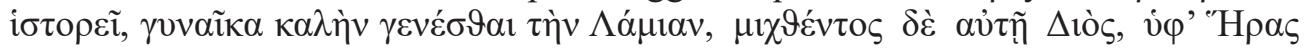

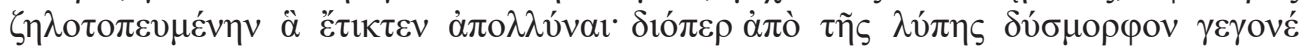

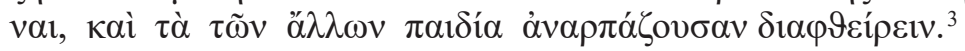

Lamia esce così dall'ambito mitografico per entrare momentaneamente in quello storiografico; Duride di Samo ne parlerebbe nella sua opera Libyaka. ${ }^{4}$ L'apparente

\footnotetext{
1 Gli scoli presi in esame che trattano della figura e della storia di Lamia sono i seguenti: Sch. Aristoph. Ves. $1035 \mathrm{~d}=$ FGrHist 76 F17, Sch. Aristoph. Pax 758 e Sch. Aristid. Pan 102.5.

2 Bisogna sottolineare che la prima attestazione, in ordine cronologico, è quella relativa alla commedia di Cratete dal titolo Lamia (CGF, IV, F20-26). Va da sé che si tratta di una commedia frammentaria, di cui per altro si hanno frammenti poco significativi; su quest'opera e le sue ipotetiche ricostruzioni si tornerà più dettagliatamente nel corso della trattazione.

3 FGrHist 76 F17.

4 L'opera è controversa in quanto non risulta nella produzione di Duride. Sia Jacoby (FGrHist IIIC) che Müller (FGH II) inseriscono, senza però nessuna spiegazione, il frammento che riporta tale indicazione nel secondo libro di $\tau \tilde{\omega} v \pi \varepsilon \rho i ̀ ~ A \gamma \alpha \theta$ ok $\lambda \dot{\varepsilon} \alpha$; con essi concorda anche KeBRIC 1977, senza specificarne le motivazioni.
} 
inconciliabilità di questi due ambiti è sanata dall'opera di Diodoro il quale, con un notevole sforzo, trasforma l'amante di Zeus in una figura storica dai contorni ben definiti e la rende una sfortunata regina, i cui figli non riescono a sopravvivere, la quale, per il dolore e per la sua indole malvagia, ordina di uccidere la progenie delle altre donne. ${ }^{5}$ Bisogna purtroppo scartare questa spiegazione così razionale e per molti aspetti evemeristica, a favore di un'interpretazione più complessa che restituisca a Lamia la sua dignità mitologica.

Nonostante questo, non è possibile nutrire dubbi sul suo genere; il participio aoristo femminile $\dot{\alpha} \lambda o v ̃ \sigma \alpha$, "essendo stata presa", con l' $\alpha$ finale, eliso per ragioni metriche, unito all'articolo determinativo, designano Lamia come essere di sesso femminile. Il significato del verbo fa supporre una certa violenza nel rapimento da parte di Zeus: il termine infatti ha numerose sfumature, tra cui quella di "cacciare", "prendere in proprio potere"; 6 forse Lamia, presa dal panico, si è lasciata andare ad un gesto così triviale anche nella narrazione mitica, $\mathrm{o}$, con più probabilità, Aristofane ha voluto scatenare una risata da parte del suo pubblico combinando questa figura mitologica con la madre di Cardopione, sui cui usi e costumi il lettore contemporaneo non è informato, a differenza degli Ateniesi che assistevano alla rappresentazione. Sicuramente è possibile dedurre che, sia l'autore che il suo pubblico, avessero una conoscenza tale delle vicende di Lamia da poterle richiamare alla mente attraverso l'uso di un semplice participio.

In un'altra opera Aristofane torna a parlare di flatulenze come caratteristica distintiva di Lamia: si tratta delle Ecclesiazuse, e questa volta il suo nome è declinato al maschile.

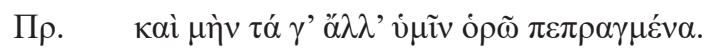

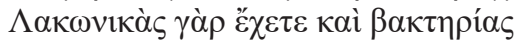

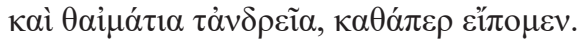

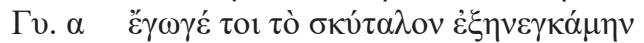

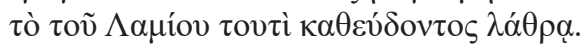

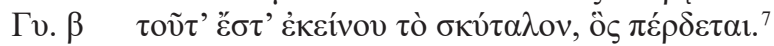

Per capire se Lamio e Lamia siano la stessa figura, bisogna ricostruire il momento comico cui appartengono questi versi; mentre Prassagora sta passando in rassegna gli abiti maschili trafugati dalle sue compagne per introdursi nell'assemblea, questa donna dichiara di aver rubato il bastone di Lamio. Potrebbe trattarsi di un nome proprio maschile indipendente, in particolare del nome del marito della donna in questione, a cui essa lo ha sottratto mentre dormiva. Ma la battuta è servita e così Prassagora, $\mathrm{o}$ meglio Aristofane, sovrapponendo immediatamente le due figure, la lancia in scena.

\footnotetext{
In realtà questo rende difficile comprendere perché l'autore del frammento ritenga necessario riportare il numero del libro delle Storie Libiche; si deve concordare con LANDUCCI GaTTINONI 2008, che ritiene che in età tardo antica le Storie su Agatocle circolassero anche sotto questo nome.

5 Diod. XX 41.

6 LSJ, s.v. $\dot{\lambda} \lambda \dot{\sigma} \sigma \kappa о \mu \alpha$.

7 Aristoph. Eccl. 73-78.
} 
Sembra proprio che la presenza, per lo meno scenica, di Lamia fosse accompagnata, a livello olfattivo, da un cattivo odore; potrebbe trattarsi di come un sistema grottesco, messo da lei in atto per salvarsi da eventuali attacchi alla sua persona. ${ }^{8}$ Lamia sarebbe quindi caratterizzata da $\delta v \sigma o \sigma \mu i ́ \alpha$, cioè da cattivo odore, qualità che condividerebbe con gli altri esseri mostruosi della mitologia greca e romana e che li contrappone ai loro avversari, gli eroi, i quali, al pari degli dèi, sono contraddistinti da $\varepsilon \dot{o} 0 \sigma \mu \dot{i}^{\alpha} .{ }^{9}$

Nella parabasi delle Vespe infine, mentre l'autore sta ricordando al suo pubblico il proprio coraggio nello scontrarsi con Cleone, troviamo attribuita a Lamia una caratteristica non presente nelle altre commedie:

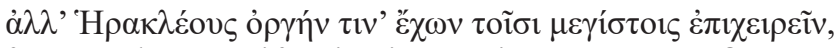

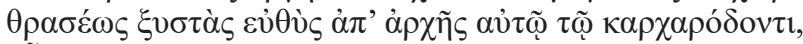

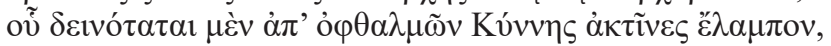

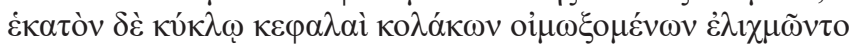

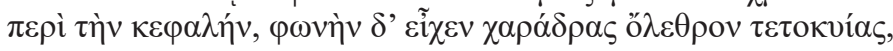

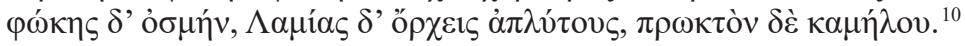

I testicoli di Lamia fanno la loro comparsa in scena come elemento caratterizzante una bizzarra e sfaccettata creatura, che sappiamo essere Cleone, composta dalle parti più disgustose o pericolose di altre creature. Per prima cosa è necessario domandarsi se questo essere, se non di sesso maschile per lo meno androgino, sia la stessa affascinante creatura che ha sedotto Zeus. Ciò è confermato dalla presenza di due elementi: il nome proprio, un genitivo femminile singolare, e l'aggettivo $\dot{\alpha} \pi \lambda v^{\prime} \tau$ ov $\varsigma_{\text {che richia- }}$ mala sfera olfattiva, ancora una volta, con una connotazione di disgusto, caratteristica che accompagna Lamia in tutte le sue comparse sceniche.

Lo scolio alle Vespe informava il lettore sulla sorte di Lamia: ella è diventata

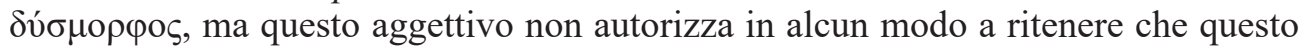
implichi un cambio di sesso o l'acquisizione parziale di attributi maschili. Suggerisce piuttosto che sia diventata mostruosa, o semplicemente deforme, ottima traduzione per questo composto che al vocabolo $\mu о \rho \varphi \eta$, "forma" o "aspetto fisico", aggiunge il prefisso negativo $\delta v ́ \sigma-$, che indica una negatività, un peggioramento o un pervertimento del concetto che accompagna. ${ }^{11}$

Neanche le altre testimonianze degli scoliasti inducono a ritenere che, in un qualche momento della sua vicenda, Lamia si sia trasformata in un uomo: lo scolio a Elio

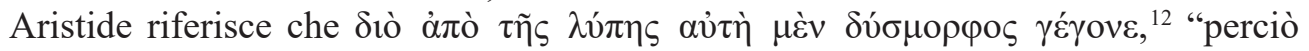

\footnotetext{
8 PADUANO - FABBro 2012.

9 Questo elemento dell'odore come caratteristica dell'alterità negativa è stato analizzato con attenzione da MAStromarco 1988 e ID. 1989. Esso risulta evidente, anticipando un verso su cui si tornerà a breve, con maggior forza nella descrizione di Cleone fatta da Aristofane nelle Vespe e nella Pace (Aristoph. Pax $753=$ Aristoph. Ves. 1035), dove per tratteggiare il 'mostro' contro cui il comico, novello Eracle, ha combattuto,

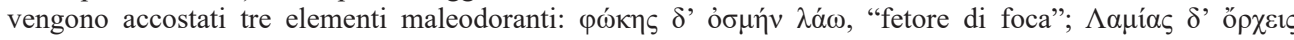

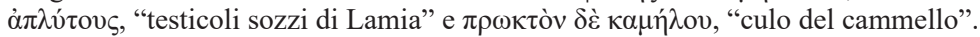

10 Aristoph. Pax 1030-1035.

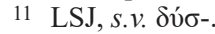

12 Sch. Aristid. Pan. 102.5.
} 
per il dolore divenne mostruosa", utilizzando il medesimo vocabolo dello scoliasta

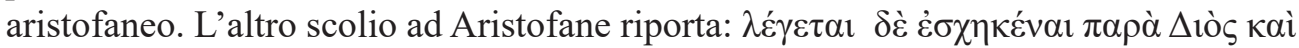

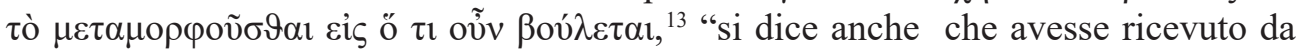
Zeus il dono di cambiare nella forma che volesse", e questa espressione indica che, volendo, Lamia avrebbe anche potuto prendere le sembianze di un uomo, ma l'essere maschio a piacimento non è paragonabile all'androginia.

Ormai è un dato di fatto: la commedia antica ridicolizza i personaggi del mito fino a renderli delle macchiette ma davvero non è spiegabile come, di fronte agli attributi così grotteschi di Lamia, gli scoliasti richiamino alla mente la nobile libica, di cui

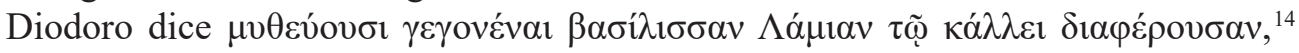
"raccontano che nacque la regina Lamia, che aveva una bellezza straordinaria".

Forse, è possibile supporre che due figure differenti, per omonimia probabilmente, siano state sovrapposte; un essere grottesco, che emette costantemente cattivo odore, ${ }^{15} \mathrm{e}$ una principessa sfortunata resa crudele e folle dal dolore per la morte dei propri figli. Questa 'seconda Lamia' potrebbe essere la protagonista di un'opera euripidea, di cui possediamo solo due frammenti e di cui non conosciamo neanche con esattezza né genere, ${ }^{16}$ né titolo. ${ }^{17}$ Nella testimonianza di Diodoro si legge:

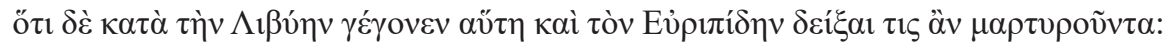

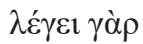

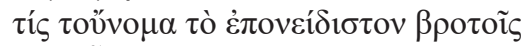

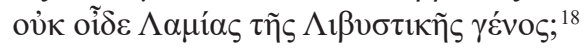

Il nome di Lamia è quindi nefasto alle orecchie degli uomini e il motivo è semplice: Lamia provoca, per gelosia e per dolore, la morte dei bambini, rapendoli e uccidendoli lei stessa nel mito o semplicemente dando l'ordine di massacrarli nella storiografia.

13 Sch. Aristoph. Pax 758.

14 Diod. XX 41.

15 Un frammento di Cratete, autorizza a supporre che Lamia fosse un personaggio prolifico per la commedia ateniese e che le sue avventure fossero ben più complesse e articolate di quanto si evince dai pochi accenni aristofanei; a lei infatti sarebbe dedicata un'intera commedia, dal titolo Lamia (CGF, IV, F20-26), i frammenti appartenenti ad essa infatti tornano sulla tematica della flatulenza (F20) ma sono presenti anche

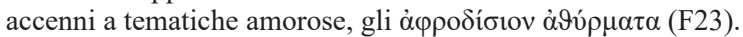

16 Esistono due tesi discordanti; la prima (MASTROMARCO - TORARo 2006) sostiene si tratti di un dramma satiresco, di conseguenza potrebbe benissimo trattarsi di un personaggio comico catapultato con i satiri nel mondo del mito 'alto' per suscitare la risate distensiva propria di questo genere drammatico. La seconda (TORTORELli GHIDINi 2000), che per varie ragioni che saranno spiegate in seguito, è quella da preferirsi, vede questo testo come una tragedia a tutti gli effetti.

17 La questione del titolo è altrettanto controversa; la denominazione Lamia è dovuta ad un frammento varroniano (Lact. Inst. I, 6, 8) che cita: in Lamiae prologo. Ma SNELL 1964, 506-507, sostenendo che il genitivo sia da riferirsi al personaggio che pronuncia il prologo e non al titolo della tragedia, attribuisce all'opera il titolo di Busiride, sulla base di un papiro di Ossirinco che riporta varie hypothesis delle opere euripidee. Busiride è il crudele figlio di Poseidone, re d'Egitto che sacrifica gli ospiti fino all'arrivo di Eracle (Hdt. II 45, 1) e quindi, come si vedrà a breve, fratello di Lamia.

18 Diod. XX 41 = TGF Eur. 922. 
Alcune fonti però,${ }^{19}$ in particolare di periodo tardo, la presentano come figlia di Poseidone e madre della prima Sibilla e non riportano mai la vicenda di Lamia e Zeus, né menzionano alcuna uccisione di bambini. La tradizione che vede Lamia come madre della Sibilla è nata in ambito corinzio e, forse, proprio questo elemento può aiutare a comprendere come ella sia diventata una rapitrice di bambini.

Eumelo di Corinto in un frammento dei suoi Korinthiakà, ${ }^{20}$ giuntoci grazie a un doppio passaggio di tradizione indiretta, ${ }^{21}$ fa narrare dalla Sibilla la contesa tra Elios e Poseidone per il dominio sull'Istmo di Corinto, contesa risolta grazie all'arbitrato di Briareo che divide la regione in due distinte sfere di competenza; in questa circo-

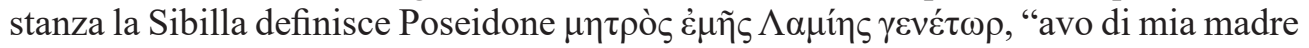
Lamia". Si può dedurre da una citazione varroniana in Lattanzio che anche la Lamia euripidea fosse collegata con la Sibilla e il mondo oracolare; Varro - in libris rerum divinarum - Sybillinos libros ita non fuisse unius Sybillae, sed - Sybillas decem numero fuisse - secundam Libycam, cuius meminit Euripides in Lamiae prologo. ${ }^{22}$

Purtroppo non si è in grado di ricostruire la fabula di questa tragedia ma è possibile, utilizzando una prospettiva 'in parallelo', provare ad avanzare alcune ipotesi in quanto, nell'opera di Eumelo, compare un altro personaggio mitologico, sempre appartenente all'epos corinzio, presentato sotto una luce completamente diversa rispetto a quella proposta da Euripide: si tratta di Medea.

Dai frammenti dei Korinthiakà, si evince che i Corinzi, dopo la morte del loro re senza legittimi eredi, abbiano chiamato Medea, nipote di Elios, da Iolco per governarli, ed ella, accettando, abbia fatto sì che Giasone, suo sposo, diventasse re di Corinto. Dell'infanticidio non vi è alcuna traccia; i figli di Medea sarebbero morti per un inganno ai suoi danni ordito da Hera. ${ }^{23}$ La regina degli dèi infatti, promettendo alla donna l'immortalità per i suoi figli, ricompensa per aver rifiutato le avances di Zeus, fa in modo che questi bambini vengano sepolti vivi nel santuario a lei dedicato.

19 Plut. de pitia 9; Paus. X 12.

20 F 8 Bernabé.

21 Il frammento di Eumelo sarebbe stato ripreso da Favorino che a sua volta è stato ripreso, proprio in questo passaggio da Dione Crisostomo; esso infatti si trova anche in Dyon. Chrys. XXXVII. Di recente Амато 2002 si è espresso contro l'inserimento di questa e di un'altra citazione (F 2 Bernabé) di Lattanzio nei Korinthiakà di Eumelo, poichè la metrica dell'oracolo riportato farebbe propendere per un'attribuzione ad uno sconosciuto poeta lirico di età classica; inoltre, sempre secondo Amato, il confronto con Pausania, che utilizza Eumelo come fonte, induce a ritenere che a cavallo tra I e II secolo d.C. circolassero soltanto versioni in prosa del poema di Eumelo. Pur accogliendo come vera questa ipotesi il punto saliente della questione, cioè la discendenza di Lamia da Poseidone, non cambia; sia che i versi siano attribuibili ad Eumelo, sia che invece vadano attribuiti ad un anonimo, testimoniano l'esistenza di questa versione alternativa nel mito di Lamia e di conseguenza nella tradizione corinzia.

22 Lact. Inst. I 6.

23 Un'altra versione riportata da Apollodoro (Apollod. I 9), purtroppo introdotta da un semplice $\lambda \varepsilon ́ \gamma \varepsilon \tau \alpha 1$, vuole che in realtà i figli di Medea vengano uccisi, nonostante la madre li avesse lasciati come supplici al tempio di Hera Acraia, dagli abitanti di Corinto. Proprio per questo motivo, secondo uno scolio di Parmenisco (Schl. Eur. Med. 264), i Corinzi avrebbero pagato Euripide ben cinque talenti perché li 'ripulisse' dell'infamia di aver assassinato dei supplici presso il tempio di Hera. In questo modo essi, liberatisi dal giogo di sovrani barbari, attraverso l'omicidio sacrilego della loro genia (questo è deducibile attraverso le maglie del racconto mitico), avrebbero commissionato il poeta ateniese affinché fissasse, attraverso la scrittura, una versione differente del mito che li rendesse innocenti. 
Ma questo è un inganno; i bambini muoiono e Giasone, una volta scoperto il fatto, abbandona la moglie e il trono e così Corinto passa in mano ai discendenti di Eolo.

A questo punto si può ipotizzare che Lamia facesse parte, come Medea, della saga pre-ellenica degli Argonauti, anche grazie alla testimonianza di Esiodo, il quale, in

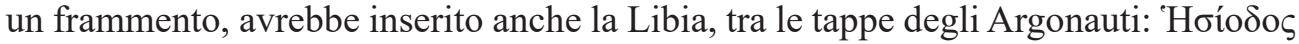

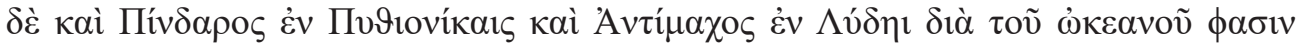

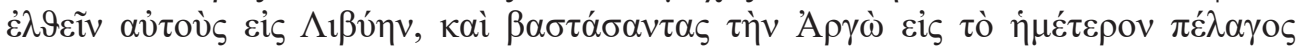
$\pi \alpha \rho \alpha \gamma \varepsilon v \varepsilon ́ \sigma \vartheta \alpha{ }^{24}$

Lamia e Medea inoltre sono caratterizzate da notevoli analogie: entrambe queste donne perdono i figli e questa perdita, in qualche modo, coinvolge la moglie di Zeus, la quale, per vendetta o per semplice crudeltà, si accanisce su di loro con la violenza o con l'inganno. Inoltre, entrambe occupano una posizione di potere in virtù della loro discendenza dagli dèi sovrani di Corinto, Poseidone e Elios, e che questo potere, esplicitamente per quanto riguarda Medea, ${ }^{25}$ ad un certo punto delle loro vicende viene loro strappato via a favore dei Corinzi 'puri'.

Adottando una prospettiva evemeristica è possibile cercare di rintracciare dietro il mito l'eco di vicende storiche realmente accadute: così questi racconti di madri non-corinzie, imparentate però con gli dèi principali della città, cui vengono uccisi i figli e in seguito perdono il potere, sono il riflesso di tensioni dinastiche, aggravate dalla presenza di stranieri, realmente avvenute nel corso della storia di Corinto. ${ }^{26} \mathrm{Si}$ potrebbe così ipotizzare che nella città di Corinto, all'epoca della formazione della polis, vi siano stati dei passaggi di potere, cui hanno preso parte anche personaggi non originari della città, magari in virtù di ipotetiche parentele con gli dèi protettori della polis stessa, ma che i Corinzi siano sempre riusciti, attraverso l'attribuzione di colpe morali e, come nel caso di Medea, dell'eliminazione fisica dei discendenti, a riappropriarsi del potere, sottraendolo ai non-corinzi.

Questa Lamia 'tragica' potrebbe essere la Lamia che hanno in mente gli scoliasti quando, al suo rumoroso e puzzolente apparire nella commedia, inseriscono un appunto per aiutare il lettore.

Ma è quasi certo che la Lamia tragica non sia l'ispiratrice della Lamia mostruosa; le testimonianze su questa sua facies sono tutte posteriori alla metà del V secolo a.C. e stonano notevolmente con Aristofane. Potrebbe trattarsi di tentativi di costruire una storia unica e personale intorno a un personaggio che in precedenza non ne era dotato, sovrapponendolo ad un altro del ciclo corinzio; Lamia infatti potrebbe essere nata con l'iniziale minuscola e al plurale.

Si è detto che Lamia fa parte di quella schiera di demoni femminili dotati di scarsa individualizzazione. Questi gruppo di figure demoniache, classificate da Belmont

24 F241 Merckelbach-West $=$ Schol. Ap. Rhod. $\Delta 259$.

25 Non si trova nelle fonti mitografiche, che distinguiamo da quelle storiografiche (quindi da Duride e Diodoro), alcuna menzione di una perdita di potere da parte di Lamia; si trova però l'indicazione di una sua discendenza reale o comunque nobiliare e siamo autorizzati ragionevolmente a supporre che il suo ritiro dalla vita sociale, dopo la morte dei suoi figli e i tormenti di Hera, e la sua trasformazione l'abbiano indotta ad abbandonare qualsiasi posizione di potere occupata in precedenza.

26 Tortorelli GHidini 2000, 13. 
come "esseri verbali", ${ }^{27}$ viene anche indicato come "Circolo di Ecate", ${ }^{28}$ e ad esso appartengono creature, solitamente di sesso femminile, la cui caratteristica comune è quella di perpetuare l'omicidio, a scopo di nutrimento o semplicemente per sadismo, con metodi brutali e predatori. Tra di esse si possono distinguere due categorie particolari; le seduttrici, che, dopo aver conquistato giovani uomini, se ne nutrono e le "child-snatching demons", ${ }^{29}$ le quali rivolgono la loro attenzione a bambini, per lo più neonati. Lamia rientra in entrambe queste categorie.

Nelle testimonianze finora esaminate si ha riprova della sua attitudine all'infanti-

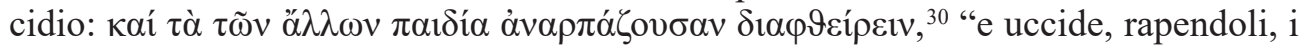
bambini delle altre", con alcune minime varianti lessicali, è ripetuto da tutti gli sco-

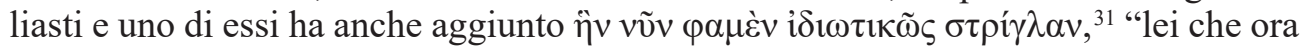
chiamiamo volgarmente strige".

$\Sigma \tau \rho i ́ \gamma \lambda \alpha \nu$ è una 'grecizzazione' del termine latino strix, una creatura metà donna e metà uccello rapace, chiamata così in virtù degli orribili suoni da lei emessi che, secondo la testimonianza di Ovidio, durante la notte salirebbe sul petto dei bambini dormienti per succhiare loro il sangue. ${ }^{32}$ L'unico modo per evitare la morte dei piccoli, avverte l'autore dei Fasti, è dare in cambio delle loro viscere quelle di un maialino, procedimento simile ad un sacrificio: Ovidio usa l'espressione hanc animam vobis pro meliore damus, ${ }^{33}$ "diamo a voi questa vita in cambio di una vita di più valore", che indica da una parte la consapevolezza l'inevitabilità dell'omicidio perpetuato dalla strigi per nutrimento, dall'altra la possibilità che esse accettino uno 'scambio' e che, in questo modo, la vita dei neonati possa essere salvata.

A questo elemento, che identifica le strigi come qualcosa di più che semplici spauracchi, si aggiunge la variabile della lussuria, in quanto esse, in alcuni casi, possono essere delle donne bellissime, perverse e dedite alla lascivia; il gesto stesso di succhiare il sangue stando sopra la vittima si trasforma in un gesto erotico, qualora la vittima sia un giovane uomo. Bisogna sottolineare quindi, ancora una volta, l'ambivalenza di questi demoni che, se da un lato sono esseri deformi divoratori di bambini, dall'altro però mostrano il loro profilo migliore quando si tratta di sedurre e portare alla morte inconsapevoli uomini. ${ }^{34}$

Esistono testimonianze che colgono Lamia proprio in questa veste di seduttrice: nel IV libro di Vita di Apollonio di Tiana, Filostrato racconta di come il filosofo sia riuscito a salvare la vita a Menippo, un suo discepolo che, dopo aver incontrato una bellissima donna sulla via di Corinto, decide di sposarla, completamente ignaro del fatto che si tratti di un'empusa.

Durante il matrimonio il filosofo rivela ai banchettanti l'identità della sposa: $\dot{\eta}$

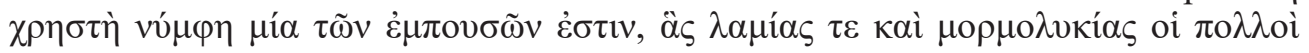

27 Belmont 1974.

28 Heckenbach 1912.

29 RYBAKova 2004.

30 FGrHist 76 F17.

31 Sch. Aristeid. Pan. 102.5

32 Ov. Fast. VI, 131-143.

33 Ov. Fast. VI, 163.

34 Perea Yébenes 2002, 267. 


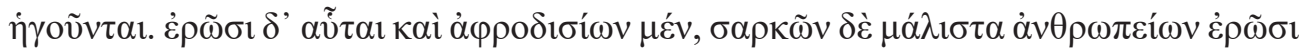

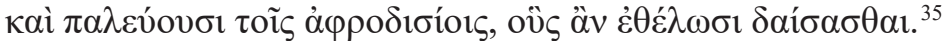

In realtà la donna di Corinto non è, quindi, propriamente una lamia, ma un'empusa. Le Empuse fanno parte anch'esse del circolo di Ecate; condividono con le altre figure demoniache una sessualità deviata e il gusto per il sangue e la carne umana ma, soprattutto, sono in grado di mutare aspetto, anche se quello che preferiscono è quello di giovani donne attraenti. ${ }^{36}$ Basti citare la bellissima donna su cui il Dioniso delle Rane vuole gettarsi a capofitto; il dio desiste dal suo proposito solo dopo che il servo Xantia gli ha fatto notare che l'attraente fanciulla sta continuamente cambiando forma e che, dulcis in fundo, ha una gamba di sterco di vacca. ${ }^{37}$

Ancora una volta vediamo ripetersi due situazioni caratteristiche di Lamia; il cattivo odore, questa volta acuito dalla presenza dello sterco, e il mutare della forma originaria. Vediamo che Lamia è passibile di una suddivisione in una molteplicità di creature antropofaghe, simili a lei ma non del tutto identificabili. L'idea che esistesse più di una Lamia circolava anche in ambito latino: il poeta Lucilio scriveva contro la superstizione:

Terriculas, lamias, Fauni quas Pompiliique
instituere Numae, tremit has, hic omnia ponit.
Ut pueri infantes credunt signa omnia aena
vivere et esse homines, sic isti somnia ficta
vera putaret, credunt signis cor inesse in aenis. ${ }^{38}$

Lamia, o meglio le lamie, sono inserite nella categoria delle terriculae, termine traducibile con spauracchi, insieme ai Fauni che le varie personalità religiose, i 'Numa Pompilii', avrebbero introdotto a Roma.

Si introduce qui un nuovo elemento caratterizzante di Lamia: ella è uno spauracchio per bambini, il cui compito è quello di spaventarli per indurli così all'obbedienza e, ovviamente, si trovano dei paralleli anche nelle fonti greche.

Diodoro nella sua razionalizzazione parla anche della facoltà straordinaria di Lamia di poter estrarre gli occhi a suo piacimento e deporli in un cestino per riposare $;{ }^{39}$ nella sua interpretazione questo "deporre gli occhi in un cestino" è una metafora dell'ubriachezza di Lamia, la quale, in questi momenti, dava a tutti la facoltà di fare ciò che volessero come se lei non potesse vederli. ${ }^{40}$ Ma conclude che questa parte, la

35 Philostr. Ap. IV 25.

36 Philostr. Ap. II 4.

37 Aristoph. Ran. 285-296.

38 484-488 Marx.

39 Sch. Aristoph. Pax 758.

40 Secondo Cusumano 2008, 52, l'insonnia perenne di Lamia sarebbe indizio di una triste immortalità; se Sonno e Morte infatti sono gemelli, come afferma Esiodo nella Teogonia (Hes. Teog. 756-759), essere sottratti al Sonno equivale ad essere sottratti alla Morte. Questo tipo di immortalità però non appare affatto desiderabile: Zeus dona a Lamia la facoltà di potersi levare gli occhi a suo piacimento per poter 'riposare' da questa condizione di eternità. Si spiega anche per quale motivo ella, senza occhi e quindi dormiente, sia innocua; nei momenti di riposo Lamia è come morta, una piccola e momentanea morte che la solleva dalle pene ma che è destinata ad avere breve durata. 
più favolistica e quindi difficile da razionalizzare, è quella che è rimasta più impressa

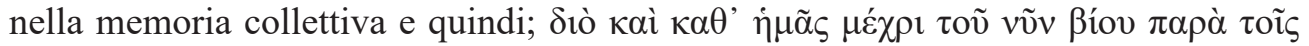

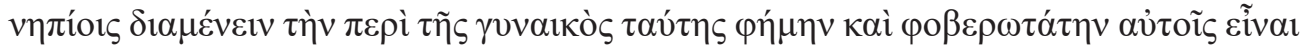

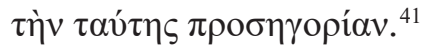

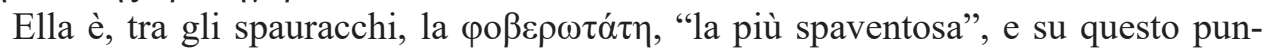

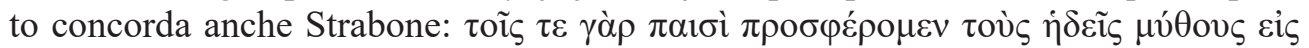

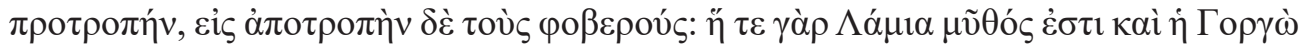

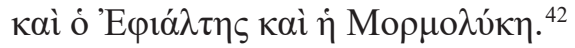

Ancora una volta si può notare come Lamia sia in buona compagnia, accostata a Gorgò e ad altri personaggi 'demoniaci', quali Mormolice ed Efialte; prima di focalizzare l'attenzione sulle implicazioni di questi accostamenti, è bene soffermarsi sul particolare degli occhi 'estraibili' di Lamia.

Il particolare è menzionato da Diodoro e dallo scolio alla Pace di Aristofane con una valenza completamente opposta: mentre nello storico è indice di estremo pericolo in quanto, quando la regina Lamia si ubriacava, dava a tutti la facoltà di compiere

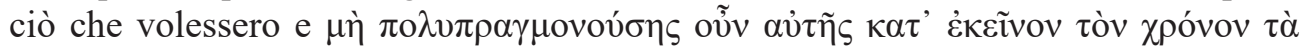

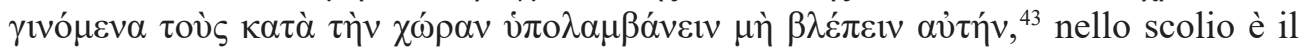
momento in cui le madri possono stare tranquille per la vita dei loro figli. Per lo scoliasta, Lamia è in grado di togliersi gli occhi per riposare e questo è un dono fattole

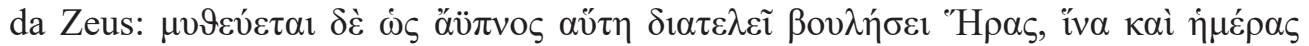

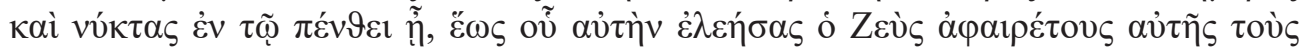

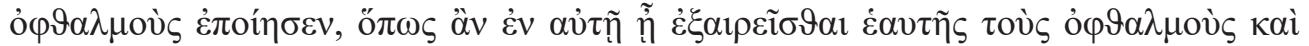

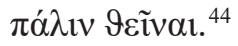

Si tratta del tema del cosiddetto "sonno del mostro", l'unico momento in cui egli non può nuocere agli esseri umani, in quanto privato della sua facoltà visiva; senza occhi un essere non può nuocere. Come ha giustamente rilevato Burkert l'occhio che fissa è uno dei principali fattori ansiogeni per l'uomo in quanto richiama alla sua mente un programma biologico ben definito: ${ }^{45}$ i predatori iniziano la ricerca di cibo attraverso lo sguardo, sguardo che, a sua volta, viene percepito dal 'cibo' potenziale il quale, sentendosi osservato, percepisce l'imminenza del pericolo. L'uomo ha memoria del momento in cui anch'egli era 'cibo in potenza'; la cultura ha manipolato questo timore e lo ha concretizzato nello sguardo maligno attraverso la credenza del malocchio, a cui, se si vuole sfuggirvi e invertire così i ruoli preda-predatore, è necessario opporre una forza maggiore, un'aggressività maschile che si concretizza nell'esibizione fallica, oppure accecare il predatore e renderlo così inoffensivo, come è accaduto per il Ciclope, il più conosciuto predatore antropofago. ${ }^{46}$

\footnotetext{
41 Diod. XX 41.

42 Strab. I 28.

43 Diod. XX 41.

44 Sch. Aritoph. Pax. 758.

45 Burkert 2003, 64.

46 Hom. Od. IX, 371-397.
} 
Così, quando Lamia depone i suoi occhi per riposare, è momentaneamente sconfitta: il suo occhio di predatrice non può fissarsi su nessun infante e in questo modo non è in grado di nuocere.

Si è visto come Lamia venga avvicinata a Gorgò, personaggio mitico, fondamentale nella saga di Bellerofonte, saga ritenuta per molti aspetti non-greca e per la precisione di ambiente semitico ${ }^{47}$ inoltre questa figura, triplicandosi nelle "Gorgoni", Medusa, Stenno e Euriale, ha anche un ruolo nella Teogonia di Esiodo, dove è annoverata tra i demoni pre-olimpici insieme alle sue sorelle. ${ }^{48}$

Il nome Mormolice, invece, è conosciuta anche come Mormò, nome etimologi-

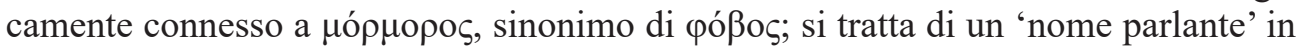
quanto potrebbe essere reso come "colei che provoca paura" o, addirittura, potrebbe essere la paura personificata. A questo nome si accosta anche il termine $\lambda v \kappa o ́ s$, che autorizza a ritenere Mormolice non solo un essere vorace e predatorio ma anche dedito all'antropofagia e alla necrofagia, ${ }^{49}$ quindi connesso con il mondo degli Inferi e delle apparizioni spettrali, come l'animale da cui prende il nome.

Anche il nome di Gorgò è etimologicamente derivato dalla sua attività principale; deriva infatti da $\gamma o \rho \gamma o ́ s$, traducibile con "selvaggio", "feroce", a cui si aggiunge il suffisso $-\omega$, molto prolifico, secondo Chantraine, nella formazione di nomi femminili e di forze personificate. ${ }^{50} \mathrm{~A}$ questo punto quindi è bene notare come persino Empusa e Lamia siano nomi parlanti: il primo si collega al verbo $\kappa \alpha \tau \varepsilon \mu \pi \alpha ́ \zeta \omega$, "sorprende-

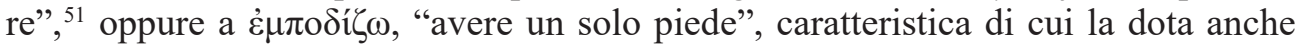
Aristofan, descrivendola come un essere dotato di una gamba di bronzo e una di sterco, oppure "ostacolare", 52 azione che le è propria in quanto Filostrato e i suoi discepoli la incontrano lungo la strada, dove lei, terrorizzando i viaggiatori, impedisce loro di continuare.

Il nome Lamia, quindi, racchiude l'azione principale attraverso la quale è stata inizialmente immaginata e di conseguenza identificata; il suo nome sembra diretta-

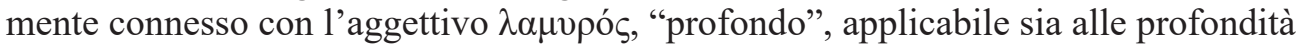
marine ma anche agli esseri viventi con il significato di "vorace". ${ }^{53}$ La stessa radice $\lambda \alpha \mu$ - è condivisa anche da altri lemmi come $\lambda \alpha \mu \mu$ ó $\varsigma$, "gola", $\lambda \alpha \mu \mu \alpha ́ \zeta \omega$, "sono vorace",

47 Molti elementi di questa saga mitica hanno indotto WEST 1997, 365-367, a ritenere che si tratti di un racconto elaborato in area semitica e penetrato poi nel mondo greco. Questi elementi sono, in particolare, l'etimologia del nome dell'eroe, da Baal, il dio fenicio, l'uccisione della Chimera e la presenza della scrittura, altrimenti sconosciuta al mondo omerico (Hom. Il. VI 155-205). Se così fosse anche la Gorgone potrebbe essere frutto di una rielaborazione greca di un demone semitico; ipotesi affascinante perché potrebbe, come vedremo, applicarsi anche al caso di Lamia.

48 Hes. Theog. 274-281.

49 Il lupo, assieme al cane e al cavallo, condivide nel mondo greco la fama di animale legato al mondo degli Inferi (PISANo 2013); essi infatti si nutrono dei cadaveri dei guerrieri caduti sul campo e le loro caratteristiche, sia visive come ad esempio lo sguardo del lupo, che sonore, soprattutto per quanto riguarda i cavalli con il rumore vibrante degli zoccoli, formano dei modelli per la descrizione dei mostri dell'infanzia analizzati nel presente lavoro.

50 Chantraine 1968, 78-96.

51 Carnoy 1957, s.v. "Empousa".

52 Etym. Mag. s.v. "Empousa".

53 Chantraine 1977, s.v. $\lambda \alpha \mu$ $\mu$ ós. 
o $\lambda \alpha 1 \mu$ o $о \mu \varepsilon \varepsilon \omega$, "taglio la gola"; si tratta di attività orali concernenti il nutrimento e questo autorizza a supporre che la caratteristica principale di Lamia è quella di essere una divoratrice. ${ }^{54}$

Questa stessa radice, $\lambda \alpha \mu-$, potrebbe essere condivisa, seppur soggetta ad apofonia, da un'altra categoria di demoni, i lemures latini. Purtroppo su queste creature le notizie sono scarse e la più antica risale alla prima età imperiale con Ovidio; egli, nei Fasti, descrive un rituale, i cui elementi costitutivi sono decisamente arcaici, compiuto dal pater familias allo scopo di 'riscattare' la sua casa dai fantasmi dei suoi antenati. ${ }^{55}$ L'etimologia individuata da Ovidio per i lemures risale a Remo, il cui fantasma, apparso ai genitori adottivi, ha istituito per primo la festività dei Remuralia, poi aspera mutata est in lenem tempore longo littera, ${ }^{56}$ e il nome si sarebbe tramutato in Lemuralia.

I lemures quindi sarebbero qualcosa di ben più pericoloso e inquietante dei fantasmi familiari della tradizione ovidiana; il fatto stesso che esistesse un rituale preposto a placarli indica che essi erano entità potenzialmente negative. Se l'etimologia fosse corretta è possibile supporre si trattasse di demoni antropofagi e divoratori con cui i Romani dovevano necessariamente convivere: prima di essere Manes paterni essi, con molta probabilità, erano creature spaventose contro le quali era necessario tutelarsi. L'essenza di Lamia quindi sarebbe stata introdotta nel mondo latino e successivamente 'romanizzata', processo che ha comportato lo slittamento di genere.

Ma anche se lessici autorevoli ritengono assodato questo processo di 'romanizzazione' di Lamia, ${ }^{57}$ già Chantraine aveva sollevato dubbi al riguardo. ${ }^{58}$ Ovidio potrebbe non aver sbagliato indicando la radice di lemures in remures. Di tale opinione è stato anche Édon che, nel XIX secolo, si è dedicato ad uno studio approfondito del carme dei Fratelli Arvali, in cui, nei versi comunemente ritenuti un'invocazione ai Lares, identificava, veste di entità demoniache da scacciare, i Remars, creature all'origine dei Remuralia. ${ }^{59}$ Nonostante 1'ipotesi sia molto affascinante, il testo dei

54 Si segnala anche l'ipotesi, avanzata da BRILL 1990, 130, secondo la quale l'etimologia di Lamia deriverebbe dal verbo $\lambda \alpha ́ \omega$, "guardare", verbo utilizzato principalmente con un senso di aggressività; alla base di questa interpretazione troviamo l'Inno Omerico a Hermes in cui, questa voce verbale, viene utilizzata per definire lo sguardo dell'aquila (Hom. hymn. XVIII 359-360). Su questa sfumatura di significato da attribuire a $\lambda \alpha ́ \omega$ concorda anche CHANTRAINE 1977, s.v. "Lao". Quest'ipotesi etimologica avvalora le tesi finora esposta designando, ancora una volta, Lamia come una creatura predatoria e divoratrice di uomini; in realtà preferiamo considerare 'madre etimologica' del nostro personaggio la radice $\lambda \alpha \mu-$, pur riconoscendo come molto suggestiva l'ipotesi di Brill.

55 Ov. Fast. 429-444.

56 Ov. Fast. 480.

57 In uno studio sulla figura dei fantasmi nel mondo greco-romano, STRAmaglia 1999, ha sostenuto che tutte quelle entità conosciute in età tardo repubblicana come fantasmi, quindi Manes, Larvae e Lemures, fossero in origine demoni di provenienza italica o etrusca; i Manes sarebbero stati divinità dell'oltretomba, forse spiriti psicopompi, le Larvae degli spiriti che attraverso la possessione inducevano gli esseri umani, in particolare le donne, alla pazzia e i Lemures sarebbero demoni antropofagi di origine greca giunti a Roma attraverso quella che Stramaglia definisce "la mediazione etrusca". Per i Lemures vd. anche HiLd 1900.

58 Chaintraine 1968, s.v. "Lamyros".

59 L'ipotesi di ÉDON 1882, 296-321, si basa sulle difficoltà oggettive di traslitterazione dal corsivo al capitale lapidario con cui è iscritto il carmen (CIL VI $2104=I L S 5039)$. Così i versi 2 e 3 (Neve lue, rue, Marmar, sins incurrere in pleores! Satur fu, fere Mars, limen sali, sta ber ber!) descriverebbero in realtà 
Carmen dei Fratelli Arvali è, oggi, dato per certo e i fantomatici Remars non vi fanno alcuna apparizione. ${ }^{60}$

L'unico merito che è possibile riconoscere a questo studio è quello di aver avvalorato l'ipotesi etimologica ovidiana: sarebbero cioè esistiti degli esseri sovrannaturali Remures o Remars, i futuri Lemures, portatori di morte in virtù della loro dipendenza dalla radice indoeuropea *mar, identificata come la radice dei termini connessi alla morte, quali ad esempio mors o morbus. Proprio per questo motivo, quindi, sarebbero necessario un complesso rito di purificazione e protezione, che prende il nome dalle creature che si vuole rendere impotenti: i Remuralia.

Di conseguenza i Romani conoscevano queste entità antropofaghe, verosimilmente maschili, chiamate Remars, prima ancora di entrate in contatto con la cultura greca, successivamente poi, venuti a conoscenza dell'essenza principale di Lamia, quella cioè di divoratrice, e della sua caratteristica di essere dotata, in alcuni casi, di entrambi gli attributi sessuali, potrebbero aver sovrapposto le due figure e aver trasformato la " $\mathrm{R}$ " iniziale in una " $\mathrm{L}$ ".

Potrebbe utile rintracciare nelle credenze spirituali, o demoniache, di popoli entrati in contatto con quello greco, la presenza o meno di entità antropomorfe e antropofaghe, simili a Lamia: rivolgendoci all'area mesopotamica troviamo una figura, Lamashtu, ${ }^{61}$ che condivide con essa molte caratteristiche essenziali, in primis l'uccisione di bambini e l'antropofagia.

il rituale dei Lemuralia e questa tesi si basa su tre punti principali. Il primo riguarda l'espressione NEVE, che sembrerebbe introdurre un imperativo negativo, ma che secondo lo studioso dovrebbe essere reso col corsivo $h i \mathrm{mi}$, in latino arcaico e his mihi, in latino classico. Si passa poi al gruppo di lettere LVAERVE (che presenterebbe due varianti nel corso del teso: LVER VE e LVUERVE); quest'ultimo andrebbe trascritto come lua fave; la voce verbale lua però non ha nessuna spiegazione, così Edon sceglie di assimilarlo alla forma del congiuntivo indicativo luam; date queste considerazioni si avrebbe in latino classico: His luam mihi fabis, pago per me con queste fave. Bisogna ora rivolgersi al gruppo MARMA, MAR MuR e MARMA e EVREREMARS, FVFEREMARS, FVFEREMARS. Per prima cosa Marmar, l'antico nome italico del dio Marte, sarebbe stato un'entità un po' diversa prima di venire assimilato al dio greco della guerra in virtù della dipendenza dall'ipotetica radice *mar-, qui raddoppiata. Sarebbero quindi affini alla greca Mormolice, conosciuta anche con il nome Mopú́v. Di conseguenza Marmar, prima di incontrare Ares, sarebbe stato anche lui un membro del circolo di Ecate. Nel secondo gruppo l'espressione FVFE (analoga a EVRE, per uno scambio di consonanti F-E e F-R), avverte Édon, potrebbe avere un altro significato: la F sarebbe stata scelta a discapito della C alterando il significato fuce Remars, dove fuce sostituisce fuge del latino classico. Si riporta, per completezza e a corredo di questa nota, la resa grafica del testo così come viene riportata da Édon:

(...)NEVE LVAERVEMARMa SINS IN* CVRRERENLDLEORES NEVELVER VEMAR

NSIN CVRREREINTL $f$ ORIS NEVELVERVE MARMAR* SERPLNCVRREREINP $e$ EOIVS
SATVR* EVREREMARS LIMEN

ESTABERBER SATVR* FVREREMARS (...).

Purtroppo, per quanto convincente e metodologicamente ineccepibile, tale ipotesi interpretative va scartata in virtù della ormai certa traslitterazione del testo.

60 Si rimanda al lavoro del francese SCHEID 1998.

61 Il nome Lamashtu è in lingua accadica e corrisponde al sumero Dimme. Nella presente ricerca, ogni qualvolta sarà necessario presentare una divinità del mondo mesopotamico, sarà utilizzato il nome accadico $\mathrm{e}$, tra parantesi, verrà indicato il corrispondente sumerico, ad eccezione di Pazuzu che non è conosciuto dalla cultura sumera. 
Secondo la mitologia mesopotamica, sumerica e babilonese nello specifico, Lamashtu (Dimme) è un demone femminile di aspetto ibrido, ${ }^{62}$ figlia di Anu (An), dio del cielo a cui si deve l'atto creativo del mondo, e sposa del demone Pazuzu, re dei demoni dell'aria. Essa è responsabile della morte dei neonati e delle donne incinte. La sua azione principale, riportata nel cosiddetto Labartu text ${ }^{63}$ consiste nell'uccidere i bambini mentre si trovano ancora nel grembo materno: "she sweeps the innards of the pregnant women, she tears the children out of the pregnant". ${ }^{64}$ Ma i bambini, una volta nati non sono comunque al sicuro, il seno di questo demone dà del latte avvelenato e, in questo modo, essa uccide i neonati che riesce a rapire, con l'inganno o chiedendoli direttamente alle madri come in questo testo: "Bring me your children, that I may suckle them, and your little girls, that I may be their guardian; to the mouth of your little girls I want to give the breast". ${ }^{65}$

Lamia potrebbe essere, come sostiene D. R. West, ${ }^{66}$ un'evoluzione greca di questo demone: il fonema -shtu potrebbe essere stato abbandonato, perché sconosciuto alla lingua greca, e sostituito con una desinenza di prima declinazione femminile con l'aggiunta, tra $\mu$ e $\alpha$, dello i per assonanza con il termine $\lambda \alpha \mu$ ós, gola. Ma Lamashtu è comunque di sesso femminile; se anche Lamia fosse una sua diretta filiazione, $\mathrm{i}$ testicoli potrebbero essere un apporto della cultura greca, in quanto nessuno dei testi a nostra disposizione fa menzione di attributi maschili.

Esiste però nella cultura mesopotamica un altro demone preposto all'uccisione degli infanti tramite l'allattamento al proprio seno, si tratta di Ĝallû, demone sicuramente di sesso maschile, in quanto il suo nome significa "grande uomo"; potrebbe allora darsi che i genitali maschili di Lamia non siano una semplice invenzione aristofanea, ma un 'prestito' di Ĝallû. ${ }^{67}$

62 L'immagine di Lamashtu è molto suggestiva e composita. La forma del suo corpo ricorda solo vagamente una figura femminile principalmente per la presenza dei seni da cui prendono il latte due bestie, un cane e un maiale; per il resto presenta una testa leonina con orecchie d'asino, mentre la parte inferiore del corpo è costituita dalle zampe di un uccello rapace e, solitamente, regge due serpenti, che possono essere in alcune rappresentazioni bicefali. Le immagini di Lamashtu si trovano principalmente su placche metalliche di epoca neo-assira; per esemplificazione vd. placca di protezione in bronzo contro il demone Lamashtu, Muséé du Louvre, inv. AO 22205.

63 Sotto il nome di Labartu si nasconderebbe la figura di Lamashtu (UNGNAD 1925) e, di conseguenza, gli incantesimi, volti a proteggere le donne incinte e i neonati, contenuti nel Labartu text, sarebbero volti a scongiurare proprio l'influsso malevolo di Lamashtu.

64 Labartu Text 2.3.29-34 (trad. di D. R. West).

65 AO 6473, Rev. 19-20 (trad. di D. R. West).

66 WeSt 1991, 368.

67 Ĝallû in realtà, oltre ad aver donato alcuni suoi attributi a Lamia avrebbe avuto, secondo WEST 1991, una filiazione diretta nel mondo greco. Troviamo infatti un personaggio parallelo a Lamia, la cui storia è molto simile, eccezion fatta per l'assenza di elementi divini: si tratta di Gello, una ragazza di Lesbo, che sarebbe morta senza aver avuto figli e questo rimpianto l'avrebbe spinta, sotto forma di fantasma, ad appagare il suo senso materno rapendo i figli delle altre donne (Suid. s.v. "Gellous" = Zenod. III 3). La stretta dipendenza tra queste due figure è anche di tipo etimologico; mentre per Lamia esistono plausibili etimologie in lingua greca per Gello è impensabile un rapporto con $\gamma \varepsilon ́ \lambda \omega \varsigma$, "risata", unico termine che ne condivide la radice. Molto più plausibile è il passaggio ipotizzato da West in cui il nome Gallu, attraverso alcuni passaggi intermedi (Ĝallû > ${ }^{*}$ Gallō $\left.>\Gamma \varepsilon \lambda \lambda \omega / \Gamma \imath \lambda \lambda \omega ́\right)$ si sarebbe trasformato in Gello per facilità di pronuncia e popolarità della desinenza -w per i nomi propri femminili. 
Secondo una teoria di 'filiazione pure', che è sempre accettare con riserva, il mondo greco sarebbe venuto a conoscenza di questi due esseri antropofagi e li avrebbe rielaborati in un unico personaggio che rispondesse alle loro esigenze culturali. Ma le figure di demoni antropofagi femminili sono onnipresenti nelle culture e, soprattutto quando il loro nutrimento principale sono bambini in fasce, rispondono a un'esigenza fondamentale: giustificano, biologicamente parlando, le morti improvvise di neonati attraverso la loro presenza di 'avvelenatrici', rendendo così marginalmente controllabile un fenomeno davanti al quale si manifesta tutta l'impotenza dell'uomo. Esse rispondono inoltre alla paura ancestrale dell'uomo di essere divorato da entità contro le quali non può combattere ad armi pari, forse ricordo permanente del rischio reale, in tempi remoti, di essere divorati. D'altra parte queste figure vengono rielaborate alla luce di paure sociali: se consideriamo l'impalcatura mitologica costruita sopra questi demoni, impalcatura che li vede come madri private dei loro figli, uccisi una volta nati, oppure come donne a cui è stata impedita la gravidanza, codificano, come ha sostenuto Johnston, ${ }^{68}$ il comportamento socialmente corretto per una donna greca. La cultura infatti impone loro di generare e realizzarsi come madri, qualora questo non possa accadere, anche per volontà d'altri, esse sono condannate a cercare eterna vendetta o soddisfazione di un istinto materno non pienamente realizzato: esse si trasformano così in demoni e vanno ad unirsi al già ricco circolo di Ecate.

Non a caso tutti questi demoni, a cui si può attribuire tranquillamente il nome di 'mostri dell'infanzia', condividono una storia simile; giovani fanciulle vergini morte prematuramente oppure madri incapaci di procreare. Esse si collocano così 'al di fuori'; non sono più in grado di tornare alla loro condizione precedente, quella di bambine, ma neanche di passare allo stadio successivo che è quello di donne e madri. Questa condizione di 'liminarità' permanente è alla base della loro capacità di metamorfosi; queste donne non hanno un posto fisso nel corpo sociale e di conseguenza non hanno neanche una forma fissa che permetta di individuarle e collocarle in un posto stabilito.

Non è facile risolvere la controversia sull'essenza di Lamia: o si tratta di un "child-snatching demon" a cui ad un certo punto è stata attribuita una storia e una dignità mitica, oppure è un personaggio mitico-politico della saga di Corinto, degradato a divoratore di infanti in seguito a un mutamento ai vertici politici della sua città natale. La prima ipotesi è preferibile, che si tratti cioè di un demone ctonio antropofago, attorno al quale, col passare del tempo e, soprattutto, con il trionfo delle entità olimpiche sulle caotiche figure mitologiche legate alla terra, sia stata imbastita una vicenda mitica. Questo 'trionfo olimpico' non è stato certamente indolore per le entità preolimpiche in quanto esse sono state degradate a 'spauracchi'; si è visto

68 JoHNSTON 1999, 161-203. Il lavoro di Johnston si concentra sui cosiddetti "Restless Deads", i fantasmi, in età arcaica e in età classica e le loro relazioni con il mondo dei vivi attraverso un approccio antropologico. In quest'ottica sostiene che dietro a questi demoni rapitori di bambini si nasconda sempre una donna umana che, privata dei figli, cercherebbe vendetta, in modo da stabilire così il paradigma normativo, valido per tutta la società greca, secondo il quale il fulcro della vita di una donna è la maternità, e il matrimonio ovviamente, e qualora ne venga privata non possa accedere all'Oltretomba. Questa ipotesi è affascinante ma risulta difficile vedere come 'primaria' la storia di Lamia che perde i figli per la gelosia di Hera, rispetto al semplice demone antropofago rapitore di bambini. 
come per Strabone o Plutarco le vecchie potenze demoniache, come Lamia, Gorgò o Mormolice, siano semplici mostri con cui spaventare i bambini e istruire gli adulti.

Secondo Giallongo, ${ }^{69}$ la vita degli individui di sesso maschile era costretta a confrontarsi continuamente con spaventosi mostri di sesso femminile: durante l'infanzia essi venivano pedagogicamente terrorizzati attraverso le storie di queste creature che rapiscono, mordono e divorano bambini indisciplinati, da adulti invece devono sconfiggere e uccidere questi mostri, come nel paradigma mitico di Perseo che taglia la testa alla Gorgone, e riportare ciò che c'è di ferino nell'essere umano, soprattutto di sesso femminile, sotto il controllo disciplinato dell'uomo, questa volta di sesso maschile, controllo che farà nascerà qualcosa di utile e vantaggioso per l'eroe, al pari del cavallo alato, nato dal sangue zampillante di Medusa. ${ }^{70}$

Il potente demone così diventa meno temibile: questo processo potrebbe aver trasformato Lamia in quel grottesco personaggio comico che fa sentire la propria presenza attraverso il cattivo odore. Esso potrebbe aver anche dato vita ad una differente versione della storia in cui Lamia, in veste di spauracchio divora-bambini, sia stata catturata e punita per i suoi crimini: quest'ipotesi nasce da una lekythos del pittore di Beldam, o pittore della Megera (V sec. a.C.), ${ }^{71}$ conservata nel Museo di Atene.

Halm-Tisserant, che di recente ha studiato in modo approfondito questa cerami$\mathrm{ca},{ }^{72}$ sostiene che essa vada inserita in una corrente del tutto particolare, sviluppatasi tra il 550 e il 470 a.C., da lei denominata "courant truculent", in cui gli artisti, spinti anche dalle scene sempre più violente che il teatro portava sul palco, si dedicano alla rappresentazione di torture, aggiungendo dei tratti caricaturali. ${ }^{73}$

Questa lekythos a fondo bianco si inserisce perfettamente in questa corrente. Essa raffigura una donna, legata nuda ad un albero di palma, che viene torturata da quattro satiri mentre un satiro anziano appoggiato ad un bastone, sulla destra della scena, guarda lo spettatore. La donna ha dei tratti anatomici fortemente caratterizzati, identificabili come etnici e in particolare come 'pigmei': le labbra sono molto pronunciate, il seno è molto sviluppato, il ventre è rigonfio e la colonna vertebrale presenta una deformazione, forse una lordosi. L'identità della prigioniera non è chiara: è stata identificata come una donna vecchia e brutta, una megera appunto, e questo è valso al pittore il suo nome convenzionale ${ }^{74}$ tuttavia non è una spiegazione sufficiente.

69 Giallongo 2012.

70 Secondo VERnAnt 2011, Lamia, Empusa e Gello sarebbero la trasposizione nel mondo dell'infanzia della Gorgone; PISANo 2013, 71, aggiunge che come essa pietrifica gli adulti, le sue compagne 'pietrificano' metaforicamente il bambino indisciplinato, il quale è momentaneamente uscito dall'ordine sociale, per sanzionarlo e riportarlo nei confini per lui stabiliti dalle balie che sono portavoce delle norme sociali.

71 BEAZLEY 1956, nº 292.

72 Halm-Tisserant 1989.

73 All'interno di questa corrente troviamo, oltre le opere del pittore di Beldman, il qualeera dotato di una particolare maestria nel dipingere scene di supplizi, anche altre ceramiche; ad esempio il cratere Dümmler (il cratere si trova nella collezione del Musée du Louvre, inv. E632.), conservato al Louvre, che raffigura una scena di esposizione alla gogna, e uno skyphos beota (Museo Archeologico Nazionale, inv. 442), sempre al Museo di Atene, in cui un artigiano, sospeso in aria grazie ad un elaborato sistema di carrucole, subisce violenza (HALm-TisSERANT 1989, 79).

74 Haspel 1936. 
Nonostante la donna sia di origine africana, le torture a cui viene sottoposta sono prettamente ateniesi: si tratta della pena capitale dell'apotympanismos,${ }^{75}$ un supplizio particolarmente doloroso e infamante in quanto i condannati venivano legati, spesso con catene o elementi metallici, ad un palo alle porte della città. In questo modo essi venivano esposti alla fame, alla sete, alle intemperie e ai morsi degli animali selvatici, supplizi cui potevano essere aggiunte altre torture, che solitamente consistevano in frustate, ustione dei genitali e strappo della lingua; infine la vittima veniva uccisa con un colpo di pestello alla nuca. ${ }^{76}$

Il pittore di Beldam, mediocre nelle pitture decorativi ma eccellente nel rappresentare i supplizi, ${ }^{77}$ ha deciso di raffigurare proprio questa scena ma non è facile comprendere perché abbia raffigurato una donna africana vittima di un supplizio ateniese.

Per prima cosa si deve considerare che l'iconografia dell'apotympanismos era molto familiare agli Ateniesi, grazie anche alla sua presenza nel mito ${ }^{78}$ talmente familiare da essere portata più volte sulla scena teatrale, sia tragica che comica; ${ }^{79} \mathrm{a}$ questo punto allora bisogna rileggere l'affermazione di Filocleone alla luce di questi elementi, in particolare il participio $\dot{\alpha} \lambda$ oṽ $\sigma(\alpha)$. Il verbo ha anche una sfumatura, per così dire, "giudiziaria", quindi "essere condannato"; forse Aristofane non si riferisce tanto al rapimento di Zeus ma a un momento, su cui purtroppo possiamo solo avanzare ipotesi, in cui gli Ateniesi sarebbero riusciti a catturare Lamia e condannarla a morte e, nel V secolo a.C., questa versione sarebbe stata portata sulla scena attraverso uno spettacolo comico o un dramma satiresco.

Esistono, ad onor del vero, anche altre chiavi interpretative che non identificano con Lamia la donna torturata nella scena. Buschor ritiene che si tratti della raffigurazione dell'Isola delle Scimmie o dei Satiridi, ${ }^{80}$ descritta nella Periegesi di Pausania: ${ }^{81}$ il periegeta riferisce il racconto del navigatore Eufemo di Caria, il quale, durante una

75 Cantarella 2005, 77-81.

76 All'apotympanismos come pena capitale fanno riferimento anche Duride di Samo (FGrHist 76 F67), Senofonte (Xen. Hell. III, 3, 11), Lisia (Lys. XII 56), Demostene (Dem. Chers. 61 e Dem. Philipp. III 61) e Plutarco (Plut. Per. XXVIII); in realtà esistono anche altre interpretazioni di questo supplizio che tendono a considerarlo come l' uccisione del condannato mediante bastonate (CANTARELLA 2005, 341-342).

77 Resta ancora senza spiegazione una sua opera, conservata al Museo di Atene, e soprannominata "lekythos dei pirati", in cui vengono rappresentate le torture inflitte ad un gruppo di prigionieri da dei non meglio identificati pirati (Atene, Museo Archeologico Nazionale, inv. 487).

78 Secondo CANTARElla 2005, l'antenato mitico dell'apotympanismos può essere rintracciato nella punizione inflitta al titano Prometeo: egli, infatti, viene incatenato ad una rupe, in posizione eretta o accovacciata, ed esposto alla fiere, in particolare all'aquila inviata da Zeus affinché gli divori il fegato, il quale ricresce inesorabilmente ogni notte. Ma al di là del mito, la cui prima attestazione si trova nell'opera esiodea (Hes. Theog. 521-569, Hes. Erg. 48-54), la prima attestazione 'umana' dell'apotympanismos si trova nell'Odissea: il capraio Melanzio viene condannato ad essere legato ad una colonna $\theta v \mu \alpha \lambda \gamma \varepsilon ́ i ̈ ~ \delta \varepsilon \sigma \mu \tilde{\omega}$, “con fune straziante", e lì lasciato morire per aver tradito il suo padrone assente (Hom. Od. XXII 186-193).

79 È possibile supporre che il pubblico Ateniese fosse abituato a vedere, oltre che nella sua realtà giudiziaria, la pena dell'apotympanismos portata sulla scena teatrale. Si pensi al Prometeo Incatenato di Eschilo in cui, verosimilmente, l'attore recitava incatenato ad una colonna, oppure alla scena delle Tesmoforiazuse in cui viene parodiata l'Andromaca di Euripide; il tragico, autopromossosi a novello Perseo, corre a salvare Mnesicolo-Andromaca, condannato dopo che le donne ateniesi hanno scoperto la sua non-appartenenza al loro genere (Aristoph. Tesm. 934-946).

80 Buschor 1927.

81 Paus. I 23,6. 
tempesta, viene trascinato verso un gruppo di isole abitate da uomini scimmieschi dalle abitudini violente, e lui e la sua flotta riescono a salvarsi soltanto abbandonando sull'isola una donna straniera, a cui i Satiri fanno violenza su tutto il corpo.

L'ipotesi è affascinante ma passibile di due obiezioni, una cronologica e una iconografica: un pittore di ceramiche, attivo nel $\mathrm{V}$ secolo a.C., non può essersi ispirato a una fonte letteraria del II secolo d.C., e gli abitanti dell'isola hanno caratteri fisici scimmieschi, mentre i satiri della lekythos hanno tratti equini.

Un'altra tesi è quella esposta da Romagnoli; ${ }^{82}$ lo studioso sostiene che si tratti della rappresentazione di un dromenon antico, una sorta di dramma rituale, in cui la protagonista potrebbe essere Lamia. Il condizionale è d'obbligo perché in realtà la figura sarebbe una donna dei Cabiri; ${ }^{83}$ Romagnoli infatti, notando la massiccia presenza di queste figurine, caratterizzate da tratti etnici, ventre rigonfio e fallo sovradimensionato, nella ceramica greca, magno-greca e italica, e notando anche che essi sono sempre inseriti in contesti 'produttivi', in primis la vendemmia, sostiene si tratti di divinità minori preposte alle attività dell'uomo. Inizialmente i Cabiri avevano una loro indipendenza mitologica, in quanto esseri appartenenti a quella che lo studioso definisce "religione pre-pelasgica", termine che indica una mentalità religiosa non micenea, quindi non indoeuropea, né appartenente alle popolazioni che abitavano il territorio greco prima dell'arrivo degli indoeuropei. Ma essi si sarebbero trasformati, con l'avanzare delle divinità olimpiche, in entità tutelari dell'uomo o in servitori degli dèi maggiori e, in questa veste, entrano a far parte dei racconti mitici delle grandi potenze olimpiche: questo fa sì che i sacerdoti, dediti al loro culto, trasformino questi racconti in azioni mimate o recitate; i dromena appunto.

Sorte simile sarebbe capitata anche a quelle creature conosciute come Ninfe; la religione olimpica, che Romagnoli definisce estetizzante, le ha trasformate nelle belle creature protettrici degli elementi naturali ma, in origine, esse sarebbero state le sorelle dei Cabiri. Tutti gli 'spauracchi' femminili quindi, come Baubò e Lamia, erano inizialmente potenti quanto i loro fratelli, ma, proprio come loro, i quali sono diventati grotteschi piccoli uomini, sarebbero poi state degradate a megere o a 'ninfette'.

Nonostante ciò si deve riconoscere l'esistenza di alcuni indizi decisamente favorevoli all'identificazione di Lamia. Pensando ad un'ambientazione teatrale, la presenza di satiri, come parte viva dell'azione, e il gioco di sguardi tra l'osservatore e il satiro anziano che invita ad osservare la scena, autorizza a pensare si possa trattare della rappresentazione di un dramma satiresco. Tutti gli autori analizzati, teatrali e non, concordano inoltre sull'origine africana di Lamia, ed è così ovvio che, per la sua resa grafica, siano stati scelti quei tratti marcati che nell'iconografia identificano le popolazioni di origini africane.

82 Romagnoli 1907.

83 Nel presente lavoro il termine Cabiri è omnicomprensivo; in realtà questi esserini erano identificati con nomi diversi a seconda della loro provenienza geografica. Troviamo così i Cureti dall'Etolia e dall'Acarnania, i Cabiri da Tebe, i Telchini da Rodi e i Dattili dall'Ida. Nonostante la loro diversa provenienza essi sono sempre raffigurati con le medesime caratteristiche somatiche: uomini bassi dalla belle scura, con la colonna vertebrale deformata dalla lordosi, il ventre rigonfio, il fallo e i glutei enormemente sviluppati. 
Lamia però, per il teatro, è un ermafrodita e dovrebbe quindi presentare, anche sulla lekythos, sia gli attributi sessuali maschili che quelli femminili: il seno è presente e ben sviluppato, mancherebbe soltanto l'attributo maschile. In realtà, nonostante il colore sia scomparso in molti punti della pittura vascolare, sono ancora visibili le incisioni lasciate dal pittore: così, in prossimità della fiaccola accesa tenuta dal satiro accucciato, è possibile riconoscere un pene in erezione. ${ }^{84}$

L'ipotesi della megera crolla di fronte a questo elemento; la donna torturata dai satiri è sicuramente un ermafrodita. Di fronte a questi elementi l'unica ipotesi rimasta in piedi è quella di Halm-Tisserant: si tratterebbe di Lamia, colta in flagrante delitto, condannata morte dalla popolazione di Atene a, sentenza eseguita da un gruppo di satiri.

Esistono ancora due plausibili apparizione di Lamia, entrambe analizzate anche da Romagnoli, sulla decorazione ceramica; ${ }^{85}$ un'oinochoe,${ }^{86}$ conservata al museo di Berlino, e uno skyphos conservato in una collezione privata di Londra. ${ }^{87}$

L'oinochoe presenta un'iconografia che difficilmente si riferirebbe a Lamia: una sfinge macro e plurimastica, esce spirando fuoco da una sorta di grotta nella parte destra della ceramica; di fronte a lei una creatura femminile, il cui genere è identificabile dal seno e dai glutei, con i capelli scarmigliati, coperta di pelle non umana, che possiamo supporre si tratti di peli o squame, la affronta contrapponendo le braccia tese al respiro di fuoco. Si può supporre che Lamia, durante la sua metamorfosi, si sia ricoperta di squame, ma non si è in grado di ascrivere la lotta con la sfinge a nessun momento della sua storia mitica. Secondo Romagnoli questo mostro sarebbe una ninfa; egli identifica il tessuto epidermico come squame e di conseguenza apparterrebbe alla stirpe dei Telchini, caratterizzati da un corpo ibrido metà uomo e metà pesce, ma gli elementi a sua disposizione non permettono di classificarla come Lamia, Baubò o Mormolice. ${ }^{88}$

Lo skyphos presenta invece meno problemi in quanto raffigura una donna, completamente coperta di peli, che insegue due pigmei, uno dei quali ha già trovato rifugio su un albero di palma, mentre l'altro, abbandonati i bagagli, sta tentando di raggiungere il compagno; vicino a loro una scimmietta assiste a tutta la scena. L'ambientazione, grazie alla presenza della scimmia e delle palme, è sicuramente africana e potrebbe rappresentare la versione di Lamia dedita alla lussuria e alla perversione; per la commedia antica infatti le donne anziane e vogliose sono spesso paragonate nell'aspetto a scimmie, come riporta anche il nostro Aristofane. ${ }^{89}$

Anche se queste due testimonianze iconografiche non rappresentassero in realtà Lamia si deve ritenere, con un buon grado di sicurezza, ritenere che la lekythos del Museo di Atene la veda come protagonista.

84 Halm-Tisserant 1989, 76-77.

85 BOARDMAN 1992.

86 BEAZLEY 1956, $\mathrm{n}^{\circ} 528.44$

87 BEAZley 1971, nº 91.1.

88 Romagnoli 1907, 178.

89 Aristoph. Eccl. 1056-1057. 
Per comprendere per quale motivo Lamia, in origine generico demone antropofago, sia stata identificata, quando le si è voluta attribuire una storia personale, come africana, è necessario leggere il ‘tipo pigmeo' alla luce dell'iconografia delle sorelle di Lamia.

L'immagine della Gorgone arcaica è ben conosciuta; è caratterizzata fisicamente da una corsa a ginocchio e dalla presenza, nella maggior parte delle rappresentazioni, di ali, da un viso deformato in un ghigno mostruoso, reso più terrificante dalla lingua esposta e dai denti aguzzi, più ferini che umani, e da occhi sovradimensionati che fissano lo spettatore immobili. ${ }^{90}$ Il suo aspetto è così orrifico perché esso deve incarnare la più totale alterità.

Un altro modo, oltre all'aspetto ferino, per incarnare l'alterità è quello di scegliere una tipologia etnica completamente estranea alla tipologia principale, che immagina e rappresenta l'alterità: questa, secondo Romagnoli, ${ }^{91}$ potrebbe essere la motivazione di fondo alla rappresentazione di Lamia come 'Africana', in quanto le persone con caratteristiche etniche differenti proiettano, le une sulle altre, le caratteristiche di alterità e negatività assoluta.

Questa negatività assoluta è sinonimo, nel caso di Lamia e delle sue 'sorelle', di angoscia perenne: si tratta di un pericolo costante con il quale devono convivere non solo le madri, preoccupate di veder divorati i propri figli durante la notte, ma anche gli uomini, i quali devono prestare molta attenzione alle giovani donne che accolgono nel proprio letto, per non finire divorati. ${ }^{92}$

Si tratta, come si è già detto, di una paura ancestrale, che risale al momento in cui gli uomini correvano davvero il rischio di essere divorati dai predatori, e la genericità di questa esperienza biologica spiegherebbe come la presenza di demoni antropofagi sia diffusa in tutto l'ambiente del mediterraneo e del Vicino Oriente e quindi come questi diversi demoni, tutti caratterizzati dalla stessa prerogativa, siano nati autonomamente in ambienti diversi, senza la necessità di influenze da parte dei loro simili.

Ma l'uomo non può vivere e prosperare culturalmente se è costantemente angosciato e così ha dovuto elaborare una soluzione culturale, mitica o rituale, che eliminasse l'elemento incontrollabile, e quindi più spaventoso, da queste figure: troviamo le pratiche magiche, come le viscere del maialino, sparse all'aperto, offerta alle strigi affinché esse risparmino il bambino che avevano precedentemente deciso di divorare, oppure gli esorcismi del Labartu-Text e gli amuleti con la raffigurazione di Lamashtu, utilizzati per proteggere le donne gravide e gli infanti dalla sua malefica azione, de-

90 GLOTZ 1896.

91 Romagnoli 1907, 184.

92 Dietro a questi demoni femminili divoratori di giovani uomini, attratti attraverso il sesso, si è visto un monito sulla pericolosità di intrecciare relazioni amorose non regolamentate (CUSUMANO 2008, 55). Il piacere sessuale portato alle sue estreme conseguenze, con la degenerazione dell'antropofagia, rischia di portare il giovane uomo, che si affaccia sulla comunità degli adulti, all'annullamento fisico nel mito, e di conseguenza sociale nella realtà. Di fronte a questi elementi diventa più semplice comprendere per quale motivo Lamia sia avversata da Hera; mentre la prima simboleggia la trasgressività erotica e la distruzione che essa comporta, la sposa di Zeus rappresenta la sessualità regolamentata e soprattutto la legittima riproduzione. 
stinati a essere appesi vicino ai bambini dormienti o partati al collo dalle donne in gravidanza. ${ }^{93}$

Parallela e complementare a questa soluzione pratico-rituale, siede la cosiddetta soluzione 'eroica'; queste creature vengono sconfitte e uccise da un grande eroe, come Perseo, il quale, attraverso la loro morte, riesce a procurare, a se stesso o al genere umano, un incredibile vantaggio. Alla vittoria dell'eroe maschile succede inevitabilmente una degradazione del demone femminile in ambito culturale, che lo rende un grottesco mostro utile solo a spaventare i bambini e i meno avveduti: la paura ancestrale viene sconfitta dalla cultura e piegata ai suoi nuovi bisogni, per diventare un efficace strumento pedagogico, funzionale alla stessa.

Le entità divoratrici di bambini, infatti, sono tutte di sesso femminile e le loro storie sono riassumibili nel paradigma "gravidanza negata-sottratta": tale elemento le rende inaccettabili alla società civile, la quale le colloca così in una situazione di marginalità, situazione però che gioca a favore di quest'ultima quando esse vengono invocate, come spauracchi, dal processo educativo. Esistono, ovviamente, anche implicazioni antropologiche notevoli in quanto esse sono donne, quindi biologicamente portatrici di vita, e al contempo assassine di bambini. Le madri che uccidono, smembrano e imbandiscono un banchetto con i figli, come nella saga degli Atridi o nel mito di Filomela e Procne, negano la maternità e la rovesciano; i figli che esse hanno ospitato nel ventre e dato alla luce ritornano all'interno del corpo umano come cibo. Lamia rappresenta l'apice di questo orrore; ella ha conosciuto la maternità, ha partorito, eppure divora $\mathrm{i}$ bambini delle altre donne, quasi fosse il contrappasso dell'impossibilità di avere altri figli dopo la morte dei propri. ${ }^{94}$ Così facendo Lamia,

93 Questi amuleti si trovano anche nella tradizione ebraica e servono a proteggersi da Lilith, forse la progenitrice di tutta questa stirpe di demoni. Si tratta di un personaggio molto complesso: la mitologia ebraica vuole che essa fosse la prima moglie di Adamo, la quale, dopo aver rifiutato di giacere sotto di lui, lo abbandona per rifugiarsi vicino al Mar Rosso dove partorì figli. Jaweh, irato, decise di inviarle tre angeli perché la riportassero da Adamo ma, al suo rifiuto, decise di uccidere i suoi figli finché essa non fosse tornata da lui. L'odio per la nuova moglie di Adamo e l'invidia per la loro discendenza, fa sì che Lilith uccida tutti gli infanti che non vengono protetti dai tre angeli inviatile da Jaweh, con il quale ella ha stretto un patto, e che, durante la notte, cerchi di giacere con gli uomini che scopre a dormire soli per avere altri figli (GRAVES - PATAI 1980, 78-84). Questa versione della storia non è presente nell'Antico Testamento, in cui l'unico riferimento a Lilith come civetta si trova nel profeta Isaia (Is. 34,14), ma era sicuramente conosciuta, come testimoniano le numerose citazioni di Lilith e alle sue caratteristiche nel Talmud e nel Zohar, la raccolta di esegesi mistica del Pentateuco e del Libro di Ruth e del Sogno di Salomone. Abbiamo testimonianza del rituale apotropaico per difendere i neonati da Lilith con amuleti su cui vengono incisi i nomi dei tre angeli con il quale viene stipulato il patto e di una sorta di preghiere, contenuta nel Zohar, per allontanare Lilith dal letto coniugale. Essa deriva probabilmente da una divinità semitica dei venti, chiamata lilitu e presente nella triade demoniache cui fanno riferimento le formule magiche babilonesi. Purtroppo si tratta di un personaggio troppo complesso per esaurirne la figura in poche righe; ciò che attira immediatamente l'attenzione però è la sostanziale analogia con la storia di Lamia: dopo un rapporto "proibito" entrambe vedono morire i propri nati per mano divina, evento che le porterà ad odiare le altre donne e uccidere loro i figli. Inoltre è anche presente la componente erotica che le rende temibile anche per la comunità degli adulti.

94 Esiste, come osserva CuSUMANo 2008, 56-57, un' ambiguità di fondo tra apparato gastro-digerente e apparato riproduttivo e di conseguenza tra digestione e gestazione. Per i bambini divorati era infatti possibile, come risulta dai versi di Orazio che indicano l'utilizzo di Lamia nei motivi folkloristici, uscire interi dal ventre delle lamie: neu pransae lamiae vivum puerum extrahat alvo (Hor. Ars 340). A favore di questa tesi si può riportare il commento di Porfirione a questo passo oraziano: Haec ad infandos terrendos solet nominari. Est ergo peccatum poetae, quod tantum fingat, ut etiam deuoratur Lamiae puerum utero educat (Porph. Ars 
come tutte le 'divoratrici' nega due cose fondamentali per la società: la possibilità di crescita e maturazione a questi bambini e, di conseguenza, quella di un ricambio generazionale alla società intera. Ancora una volta questo personaggio rivela una pericolosità latente ma intrinseca per la comunità umana.

Lamia ha quindi subito sì una metamorfosi, ma essa non è dettata dal dolore o dalla facoltà donatole da Zeus, quanto, piuttosto, da una necessità culturale: della Lamia originaria non è possibile ricostruirne $\mathrm{i}$ contorni.

Proseguendo nella sua storia culturale la sua figura continua ad oscillare fino all'età tardo-antica tra il serio, aspetto però preso in considerazione solo da persone di bassa cultura, e il faceto: aspetto ben sottolineato dal mondo latino che ne conserva tale ambiguità di Lamia.

Ella da una parte viene colta soltanto nel suo aspetto di molteplicità di terricula, protagonista di storie impossibili e grottesche; Orazio usa una metafora significativa per suggerire che gli scrittori debbano mantenersi aderenti al vero nella loro narrazione, cioè che neu pransae lamiae vivum puerum extrahat alvo, "non estragga il bambino vivo dal ventre di una lamia che lo ha divorato". ${ }^{95}$ Anche per Tertulliano Lamia è la protagonista di una fiaba, una fiaba che riguarda delle torri e un pettine del Sole, lamiae turres et pectines Solis, ${ }^{96}$ di cui però non si conosce per intero lo svolgimento, anche se, dato il breve accenno che ne fa l'autore, sicuramente doveva essere molto popolare e conosciuta nel III secolo d.C.

La parte spaventosa resta nelle lamie-streghe di cui parla Apuleio, donne anziane e lascive dedite alla magia e alla vendetta, come Meroe, che maledice il povero Socrate ed orina sul suo compagno di viaggio; ${ }^{97}$ ma in realtà è una serietà degradata. Innanzitutto si tratta di una donna anziana e molto brutta, che si compiace della propria lussuria, ma comunque di un essere mortale; il mondo del mito non le appartiene più. Anche il particolare dell'orina non contribuisce a creare un'ambiente terrificante e, anzi, serve a stemperare la tensione creatasi dal rituale magico compiuto su Socrate, estraendogli il cuore dalla clavicola e raccogliendo tutto il sangue in un otre.

La Lamia spaventosa, divoratrice di infanti, è stata definitivamente uccisa; l'ultimo stadio della sua trasformazione culturale l'ha trasformata in una vecchia strega che non riesce a resistere ad un uomo e che si vendica dell'inevitabile abbandono.

Forse tutto questo perché essa ha esaurito il suo compito: dopo essere stata addomesticata in mostro dall'odore sgradevole, si è così tanto discostata dalle sue origini da non essere più riconoscibile. Gli uomini l'hanno continuata a vedere come un personaggio negativo e dotato di alterità, come comunque rimane la vecchia strega, ma all'angoscia si è sostituito il disgusto. Le sue metamorfosi, il regalo donatole da Zeus nel mito, l'hanno tanto abbassata da distruggerla.

\footnotetext{
$340,1)$. Il termine alvus è stato significativamente sostituito con uterus; il motivo del bambino divorato, poi restituito intero al mondo dopo l'uccisione del mostro, è motivo folklorico ben noto.

95 Hor. Ars 340.

96 Tert. Adv. Val. 3, 3.

97 Ap. Metamorph. 1, 5-19.
} 


\section{Bibliografia}

Amato, E. (2002): "Su due improbabili citazioni dai Korinthiaká di Eumelo (F 2; 8 Benabè = 12 Davies): un nuovo frammento lirico di autore incerto (Eschilo?) e un oracolo sibillino", Emerita, Revista de Lingüistica y Filología Clásica 70, 45-68.

BEAZLEY, J. D.

(1956): Attic Black figure Vase-Painters, Oxford.

(1971): Paralipomena: Additions to 'Attic Black-figure Vase-painters' and 'Attic Redfigure Vase-painters', Oxford.

Belmont, N. (1974): “Comment on fait peur aux enfants”, Topique 13, 101-125.

BOARDMAN, J. (1992): “Lamia”, LIMC VI, 1, 189.

BriLl, J. (1990): Lilith. L'aspetto inquietante del femminile, Genova (1 $1^{\text {a }}$ ed. Paris, 1981).

Burket, W. (2003): La creazione del sacro. Orme biologiche nell'esperienza religiosa (=Il ramo d'oro 42), Milano (1 $1^{\mathrm{a}}$ ed. Harvard, 1996).

Buschor, E. (1927): “Die Affen-Inseln”, Mitteilungen des Deutschen Archäologischem Instituts. Athenische Abteilung 52, 230-232.

Cantarella, E. (2005): I supplizi capitali. Origine e funzione della pena di morte in Grecia e a Roma, Milano (1ª ed. 1991).

CARnOY, A. (1957): Dictionnaire étymologique de la mythologie gréco-romaine, Louvain.

Chantraine, $\mathrm{P}$.

(1968): La formation des noms en grec ancien (=Collection linguistique publiée par la Société de Linguistique de Paris 38), Paris.

(1977): Dictionnaire étymologique de la langue grecque, Paris.

Cusumano, N. (2008): “Ad infantes terrendos. Sortilegi e disordine metamorfico nell'immaginario mitico greco sull'infanzia", [in] I. E. Buttitta (ed.), Miti Mediterranei. Atti del Convegno internazionale (=Acta diurna 3), Palermo, 47-65.

ÉDOn, G. (1882): Écriture et prononciation du latin savant et du latin populaire, Paris.

Giallongo, A. (2012): La donna serpente. Storie di un enigma dall'antichità al XXI secolo, Bari.

Glotz, G. (1896): “Gorgones”, DA II.2, 1615-1629.

Graves, R. - Patai, R. (1980): I miti ebraici, Milano (1 ${ }^{\text {a }}$ ed. London, 1964).

Halm-Tisserant, M. (1989): "Folklore et superstition en Grèce classique: Lamia torturée ?", Kernos 2, 67-82.

Haspel, C. H. E. (1936): Attic Black-figured Lekythoi (=École française d'Athènes. Travaux et Mémories IV), Paris.

HeCKenBaCH, J. (1912): “Ecate”, RE VII, coll. 2769.

HiLD, J. A. (1900): “Lemures”, DA III.2, 1100-1101.

Johnston, S. I. (1999): Restless Dead: Encounters between the Living and the Dead in Ancient Greek, Berkeley-London-Los Angeles.

Kebric, R. B. (1977): "In the Shadow of Macedon: Duris of Samos", Historia Einzelschriften 29, 1-99.

Landucci Gattinoni, F. (2008): “Agatocle, Ofella e il mito di Lamia (Diod. 20.41.2-6)”, Aristonothos 2, 161-175. 
MAstromarco, G.

(1988): "L'odore del mostro", Lexis 2, 209-215.

(1989): "L'eroe e il mostro (Aristofane, Vespe 1029-1044)", Rivista di filologia e istruzione classica 117, 410-423.

Mastromarco, G. - Toraro, P. (CUr.), (2006): Commedie di Aristofane, Torino, vol. II.

Paduano, G. - Fabbro, E. (EDS. \& Trads.), (2012): Aristofane, Le Vespe, Milano.

Perea YÉBenes, S. (2002): "Las Striges: mujeres-pájaro, lujuriosas, devoradoras”, [en] Id., El Sello de Dios (2), Ceremonias de la muerte. Nueve estudios sobra magia y creencias populares greco-romanas (=Signifer Libros. Graeco-Romanae Religionis Electa Collectio 9), Madrid, 233-270.

Pisano, C. (2013): "Da spauracchio per i bambini a indictio silentii. I "mostri dell'infanzia" nell'antica Grecia", [in] I. Baglioni (ed.), Monstra. Costruzione e Percezione delle entità ibride e mostruose nel Mediterraneo Antico, Roma, vol. 2, 69-78.

Romagnoli, E. (1907): "Ninfe e Cabiri”, Ausonia 2/II, 141-184.

Ryвakova, M. (2004): The Child-snatching Demons of Antiquity: Narrative Traditions, Psychology and Nachleben, Yale.

SCHEID, J. (1998): Commentarii Fratrum Arvalium qui supersunt. Les copies épigraphiques des protocoles annuels de la confrérie arvale (=École française de Rome 247), Roma.

SNELl, B. (1888): Supplementum continens nova fragmenta euripidea et adespota apud scriptores veteres reperta adiecit B. Snell, Stuttgart.

Stramaglia, A. (1999): Res inauditae, incredulae. Storie di Fantasmi nel mondo greco-romano (=Le Rane 24), Bari.

Tortorelli Ghidini, M. (2000): "Lamia e Medea in Eumelo", Vichiana 2, 3-13.

Ungnad, A. (1925): “Labartu oder Lamashtu?”, Zeitschrift für Assyriologie und vorderasiatische Archäologie 36, 108.

Vernant, J.-P. (2010): La morte negli occhi; figure dell'Altro nella Grecia Antica, Bologna, $\left(1^{\text {a }}\right.$ ed. Paris 1985).

West, D. R. (1991): “Gello and Lamia: two Hellenic Daemons of Semitic Origin”, Ugarit Forschungen XXIII, 351-368.

West, M. L. (1997): The East Face of Helicon. West Asiatic Elements in Greek Poetry and Myth, Oxford. 